\title{
El siglo de la psiquiatría alemana: ¿Cómo empezó y cómo finalizó?*
}

Uwe Henrik Peters

Universidad de Colonia, República Federal de Alemania

Recibido: 10 de enero del 2010 / Aprobado: 15 de marzo del 2010

Este artículo es una revisión histórica de la tradición psiquiátrica alemana, que tiene sus origenes tanto en la filosofía de Christian Wolff e Immanuel Kant como en el movimiento religioso denominado pietismo. El autor se refiere, en el marco de esa tradición, a los trabajos de Emil Kraepelin y Sigmund Freud, así como a la escuela fenomenológica. Finalmente, señala que en la actualidad la psiquiatría norteamericana ha desplazado a la tradición psiquiátrica alemana, incluso en la misma Alemania

psiquiatría alemana / fenomenología

\section{The century of German psychiatry: How it began and ended?}

This essay surveys the history of the German psychiatric tradition that originates in the philosophy of Christian Wolff and Immanuel Kant as well as the religious movement termed pietism. This tradition received influence from the works of Emil Kraepelin and Sigmund Freud, and the School of Phenomenology which is also discussed. The author finally claims that contemporary American psychiatry has displaced the German psychiatric tradition even in present Germany.

* Traducción del alemán por Ramón León, aprobada por el autor. Correo electrónico: U.H.Peters@uni.koeln.e 
Prácticamente en cada idioma del mundo términos como psiquiatría, esquizofrenia, psicoanálisis, psicoterapia, etcétera, son de difundido uso, e incluso personas sin mayor formación académica saben que la esquizofrenia significa una disgregación de la personalidad. Pero no todos saben que estas palabras surgieron en Alemania. Todavía menos son los que saben que los conceptos designados por ellas se desarrollaron igualmente en Alemania. Uno de los motivos de esto estriba en que para la mayoría de las personas la imagen de Alemania sigue estando, en gran medida, determinada por lo ocurrido en la Segunda Guerra Mundial y sobre todo por el Holocausto, cuyo fin sin embargo aconteció hace más de sesenta años.

En la presente comunicación se busca aclarar cómo es que los términos antes señalados aparecieron en Alemania. Se trata asimismo de precisar algunos conceptos particularmente importantes, que marcaron a fuego la centuria pasada, a la cual se le conoce también como el siglo de la psiquiatría alemana. Finalmente, se discutirá cómo y por qué este importante ciclo llegó a su fin, incluso en la propia Alemania.

País ubicado en el centro de Europa, Alemania no tiene claras fronteras geográficas. Por eso, ellas se han modificado en el tiempo como consecuencia de los avatares de la historia del pueblo alemán. Esto ha traído muchas desventajas (e innumerables desventuras per- sonales) pero también algunas ventajas. Pocas personas recuerdan que Francia y Alemania alguna vez fueron un solo país y que hubo guerras para volver a unirlas. Todavía hoy ambos pueblos rinden homenaje al mismo hombre como su fundador: Karl der Grosse en Alemania y Charlemagne en Francia, quien se coronó en la noche de Navidad del ochocientos, en Roma, como primer emperador desde los años del imperio romano. Su trono está aún en Aquisgrán, a ochenta kilómetros de Colonia.

En ambas partes del reino carolingio, Francia y Alemania, hubo en el siglo XVI un movimiento reformador religioso, al cual siguieron en el siglo XVII largas y cruentas guerras religiosas. El fin de la guerra fue sin embargo diferente. En Alemania la Guerra de los Treinta Años dio lugar en 1648 a la división religiosa del país en una parte católica y en otra evangélica $y$, con ello, a un triunfo parcial de los reformadores. En Francia, por el contrario, los reformadores -llamados hugonotes- fueron derrotados y casi exterminados. Los sobrevivientes se salvaron en gran medida en el territorio reformado de Alemania. Berlín tuvo en alguna época hasta treinta por ciento de población francesa.

En los estados reformados de Alemania la vida pudo desenvolverse de manera libre, sin tutelajes indeseados ni vigilancias aborrecibles. El horror de esta guerra superó sin embar- 
go todo lo que nos podamos imaginar hoy. La población de Alemania se redujo en un tercio en esa época. Tan pronto como lo peor fue superado, se estimuló la libertad de pensamiento. Un ejemplo. El Kurfurst del Estado de Brandenburgo solicitó al filósofo Christian Tomasius (1655-1728) un dictamen acerca de cómo podía hacer de la Universidad de Halle la mejor de Alemania. El dictamen fue muy sencillo y se puede resumir en una sola frase: "la libertad es la que da a todo espíritu una vida correcta". El príncipe siguió el consejo. Sobre la base de la entonces llamada "libertad de Halle" (Hallesche Freiheit) se convirtió esta ciudad en el asilo de todos los académicos perseguidos y la suya fue en poco tiempo la mejor y más grande de las universidades alemanas, al atraer al mayor número de estudiantes.

Los conceptos sobre los cuales se informará surgieron casi todos en Halle. Pero hubo un par de situaciones adicionales que contribuyeron de modo decisivo al surgimiento de ellos. Lutero, el gran reformador, había suprimido el celibato para los sacerdotes y él mismo contrajo matrimonio con una monja, Catalina Bora, con la cual tuvo varios hijos. Con esto se permitió a los sacerdotes las relaciones sexuales, con la limitación sin embargo de que estas ocurrieran solo en el matrimonio y de que cualquier práctica de control de la natalidad estuviera severamente prohibida.
Los pastores evangélicos vivían por lo general en medio de la pobreza. La ausencia de controles de natalidad por su parte dio lugar a familias de pastores con diez y hasta dieciocho niños, que eran educados por sus propios padres. Esta circunstancia dotó al país de intelectuales excelentemente formados. Además, las ideas de la tolerancia en el siglo XVIII favorecieron a los judíos, participantes en el desarrollo filosófico y cultural de Alemania.

Lo primero que surgió de la actual psiquiatría fue la psicoterapia. Los inicios se pueden establecer alrededor de cien años antes de la apertura de la primera clínica psiquiátrica. Ningún psiquiatra fue el responsable de que ella surgiera. La psicoterapia surgió en la cabeza de muchos. Sus raíces se pueden rastrear en la filosofía de la época y en el movimiento religioso de los pietistas. Ambos tuvieron su centro en Halle.

En primer lugar, tratemos de la filosofía. Fue considerado necesario en el siglo XVIII que todo médico, al igual que cualquier otro investigador, estudiara también filosofía. Asimismo, los filósofos y los juristas escuchaban clases de medicina. Así surgió la imagen del médico filósofo (expresada en el término der philosophische Arzt), que fue el título, a fines de ese siglo, de un libro de tres tomos Weikard (17981799). La terapia que resultó de esto se llamó terapia psíquica. Se trataba de la misma denominación que hoy tiene. 
Otra denominación fue la de cura psicológica, con igual significado. Ya en 1751 circulaba el primer texto de psicoterapia en el sentido moderno. El autor fue un médico de solo 23 años e hijo de un pastor evangélico, de nombre Johann Christian Bolten. En ese libro está la frase de que un médico que no aprende filosofía y no la aplica a sus pacientes, no es más que una farmacia viviente. La terapia de la época se refería no solo a las enfermedades mentales, sino también a las corporales, esto es cuando el estado de ánimo ha sido particularmente afectado. Bolten emplea un término de largo uso en el idioma alemán, Gemüt, que se puede traducir aproximadamente como corazón en español. En inglés y en francés no hay una palabra adecuada para él.

Hacia fines del siglo, en 1798, Marcus Herz, un filósofo judío y médico psicoterapeuta, reflexiona acerca de la definición de psicoterapia. Esto es, cómo se puede diferenciar entre la psicoterapia y los éxitos logrados a través de casuales elevaciones del ánimo (Herz 1805). La respuesta es que se aprende de experiencias casuales (lo cual siempre ha sido el caso en los medicamentos), y se aplican de acuerdo con un plan sistemático.

Desde 1803 aparece la palabra psiquiatría en lugar de terapia psíquica y los psicoterapeutas se llaman entonces médicos psíquicos o psiquiatras.

Las raíces no filosóficas de la psicoterapia se encuentran en el pietismo, un movimiento religioso del protestantismo, el cual igualmente tiene en Halle uno de sus centros. El pietismo promovía la conversión por medio del cambio de la vida interna entre la oscuridad y la luz. De esto provienen diarios muy personales e íntimos, los cuales se daban a leer a los otros. Aparecen además autobiografías y novelas autobiográficas. Por ejemplo, Karl Philipp Moritz, editor de la primera revista psiquiátrica del mundo, publicó, entre 1785 y 1790, la novela autobiográfica Anton Reiser, que puede ser considerada la primera novela psicológica de la historia (Moritz 1998). La revista antes mencionada alcanzó nada menos que diez volúmenes, y tenía como título Gnoti Sauton, "conócete a ti mismo". Estos conocimientos recónditos de los sentimientos así como de las fantasías sexuales llevarán al lector a recordar el psicoanálisis, que apareció cien años después.

Se podrá preguntar dónde ubicamos a Franz Anton Mesmer, el descubridor de la hipnosis. En realidad, no pertenece aquí: él fue un excelente observador, pero un materialista. Para él solo había una explicación física para el fenómeno de la hipnosis. De sus escritos se puede deducir que no conocía la literatura psicoterapéutica de su tiempo. Mesmer mismo quería con sus métodos solo curar trastornos funcionales, como hoy los denominaríamos: somatoform disorders. Pero, por supuesto, los psicoterapeutas se han servido de sus procedimientos. 
Al mismo tiempo, había una nueva filosofía racionalista, conducida por Christian Wolff e Immanuel Kant, en todo caso el "Kant precrítico". También Wolff enseñaba filosofía en Halle. En 1713 publicó el libro Vernunftigen Gedancken Von den Kräfften des menschlichen Verstandes und Ihrem richtigen Gebrauche in Erkäntnis der Wahrheit, hasta hoy considerado como el inicio de una nueva filosofía racionalista (Wolff 1713). Este libro comienza con una frase que inmediatamente se puede referir a la esquizofrenia: "Der Mensch hat nichts vortrefflicheres von Gott empfangen, als seinen Verstand: denn so balde er nur in demselben verrücket wird, so bald wird er entweder ein Kind, oder ärger als ein wildes Thier" ["el hombre no ha recibido nada superior de Dios que su razón: pues tan pronto él enloquece, de inmediato o se convierte en un niño o se torna más agresivo que un animal salvaje"]. Verrückt tiene el sentido de que una cosa ha sido desplazada de su lugar. En español la voz "desplazar" parece la más cercana, pero sin que esté lo Ruckhafte. Tampoco la palabra "mover" incluye ese sentido.

En 1798, Kant también publicó un libro que podemos ver como un manual racionalista de psiquiatría. Lleva el título de Anthropologie in pragmatischen Hinsicht (1980). Kant reconoce allí la locura como un trastorno de la razón y lo califica como "terquedad lógica", en latín sensus privatus.
Bajo estas concepciones tanto psicoterapéuticas como racionalistas aparecen en 1805 y 1806 en Bamberg y en Bayreuth los primeros verdaderos centros de salud -en el sentido estricto del término- en Alemania. En Francia, en cambio, la primera institución aparece recién en 1838. Este hecho apenas es considerado por la historiografía. El motivo probablemente sea que en 1806 la desgracia y la catástrofe se desplomaron sobre Alemania, cuando Napoleón alcanzó un triunfo completo contra este país, con lo cual los franceses tuvieron carta libre en Alemania. Después de mil años de existencia del imperio alemán, su emperador se vio obligado a dejar su corona. Por su parte, Napoleón, que en 1804 se había coronado emperador, buscó la restitución del reino carolingio bajo una sola corona: la suya.

En la misma época las propiedades de la Iglesia fueron confiscadas y vendidas. Los dos primeros hospitales psiquiátricos debieron ser nuevamente cerrados. Sin embargo, poco después, en Schleswig, en el norte de Alemania, fue edificado un hospital psiquiátrico que abrió sus puertas en 1820. Incluso, fue probablemente en el mundo entero el primer hospital de este tipo construido con el fin de restaurar la salud.

Schleswig Holstein pertenecía a Alemania, pero el rey de Dinamarca era duque de Schleswig. Carl Ferdinand Suadicani (1753-1824) fue quien lo construyó. 
En el resto de Alemania se edificaron numerosas instituciones psiquiátricas durante el siglo XIX. Eran, sin embargo, instituciones de cuidado de acuerdo con el modelo francés. Los médicos se denominaban alienistas, solo desde 1850 comenzaron a llamarse psiquiatras. Muy lentamente otros países siguieron esta denominación. Por ejemplo, recién en 1921 la más importante revista norteamericana especializada cambió el título de American Journal of Insanity por el de American Journal of Psychiatry.

Hasta la mitad del siglo XIX no aparecieron en Alemania conceptos nuevos y creativos en la psiquiatría. Es a partir de la segunda mitad de dicha centuria empezó a producirse algo al respecto. Esta historia es sencilla, por lo cual se deja personalizar. Kraepelin y Freud son los dos grandes nombres, dos antípodas, que se criticaban recíprocamente, pero que tienen mucho en común. Primero las semejanzas. Kraepelin y Freud nacieron el mismo año, 1856, y disfrutaron de la misma formación escolar humanística. Por ello podían leer griego antiguo y latín; a esos dos idiomas agregaban amplios conocimientos en francés, italiano, inglés, y, en el caso de Freud, también del español.

Las ideas de ambos estuvieron fuertemente influidas por sus profesores de filosofía. En el caso de Kraepelin fue el filósofo Wilhelm Wundt, quien en 1874 publicó su Grundzüge der phy- siologischen Psychologie (1874) y después, en 1879, y en Leipzig, fundó el primer laboratorio experimental de psicología en el mundo. Kraepelin, que había trabajado allí, estableció después el primer laboratorio psiquiátrico, también de todo el mundo. Siempre estuvo convencido de que sus más importantes trabajos se habían llevado a cabo allí. En esto Kraepelin se engañó a sí mismo. No fue en su laboratorio donde mostró su grandeza, sino en su capacidad para observar la conducta anormal y describirla en lengua alemana, lo cual tuvo un gran efecto en la docencia.

En el caso de Freud fue el filósofo Franz Brentano, que en 1874 publicó un trabajo parecido, Psychologie vom empirischen Standtpunkt (1874). Tanto Freud como Kraepelin tenían 18 años cuando aparecieron los libros de sus respectivos maestros. En el caso de Freud la inclinación por la filosofía fue tal que incluso pensó doctorarse bajo la dirección de Brentano.

Por lo demás, las semejanzas continúan. Tanto Freud como Kraepelin publicaron sus dos trabajos más importantes: La interpretación de los sueños (Freud 1968) y aquel en el que se trata de la dementia praecox (Kraepelin 1899), en la última década del siglo XIX. Ambos habrían de influir mucho en la psiquiatría del siglo XX y estaban ya a comienzos de esa centuria a la disposición de sus lectores.

Pero las diferencias eran también muy marcadas. Kraepelin rechazaba de 
modo decidido todo aquello que se alejara de la creencia en la capacidad explicativa de las ciencias naturales en la psiquiatría. No vaciló para ello en emplear procedimientos poco adecuados, como afirmaciones despreciativas y la ridiculización. En el caso de la teoría de Freud dos eran los temas por los que se sentía especialmente incómodo. Uno, el método freudiano de la interpretación, y el otro la presencia en el centro de la teoría del psicoanálisis de la sexualidad. Demasiado ocupado en sus propias investigaciones y en una febril productividad bibliográfica, no se tomó el trabajo de criticar directamente las ideas de Freud. De eso se encargó su discípulo Max Isserlin, judío al igual que el padre del psicoanálisis (Peters 2002a; 2002b).

Freud, por su parte, tenía como uno de sus rasgos caracterológicos más negativos el de dividir al mundo científico en seguidores y opositores. En Kraepelin rechazaba todo lo referido a la dementia praecox, para él un término muy poco elegante. Freud pensaba que las enfermedades eran dos, para las cuales proponía los nombres de 'paranoia' y 'parafrenia'. Aún más importante para él era que reconociera que la disolución de la libido con la regresión al yo era la causa de ambos. Por lo demás, la crítica del sistema kraepeliniano la dejó Freud a su discípulo Carl Gustav Jung, que tenía la ventaja de no ser judío como Kraepelin.
La discusión no ha terminado. Por el contrario, sigue durando ya más de cien años.

Al mismo tiempo que aparecieron los principales trabajos de Kraepelin y Freud hubo otros importantes desarrollos que influyeron en el curso de la psiquiatría alemana. Estos son difíciles de transmitir en otras culturas lingüísticas, especialmente la angloamericana.

Nos referimos a la idea de la estructura con dos polos. En primer lugar, formulemos la idea en el plano abstracto. Un polo es la captura de la totalidad como tal. El otro polo es el detalle, el cual ocupa un determinado lugar dentro de la totalidad. Ahora vamos a la formulación concreta: cuando escuchamos una melodía escuchamos sonidos que reconocemos. Pero lo hacemos solo por el hecho de que esos sonidos ocupan un determinado lugar y tienen determinado valor dentro de la totalidad.

Otro ejemplo: un edificio está hecho de muchos elementos, que también forman parte del mundo. En dicho edificio hay elementos más importantes $\mathrm{y}$ otros menos importantes. Si se retira algo que no es muy importante no le va a pasar nada a la edificación, pero si se retira algo estructural esta se caerá. El modelo de la estructura fue extrapolado a la psiquiatría. Desde entonces se describieron los cuadros clínicos, donde el énfasis fue puesto en las imágenes. Por ejemplo, la imagen de la melancolía. También una imagen con- 
tiene muchas pequeñas partes en un orden determinado. Por lo tanto, es posible destacar algunas partes características y dejar de lado otras. De esto hace uso por ejemplo la caricatura, en la cual solo lo más característico es presentado. Los detalles pueden ser deducidos. Casi todos los psiquiatras importantes de la época hasta Kurt Schneider, han publicado después de 1900 trabajos en cuyos títulos las palabras Aufbau, Struktur, Gestalt, Gefüge, System, Ganzheit, mehrdimensional, hacían referencia a la teoría.

A esto se agregó como complemento la más detallada descripción de los detalles psicopatológicos por medio de otra escuela psiquiátrica. Mediante estos detalles, llamados fenómenos, fue posible lograr finas diferencias diagnósticas, inclusive de cuadros psiquiátricos muy parecidos. El psiquiatra filósofo Karl Jaspers fue la cabeza reconocida de esta escuela. Poco antes de la Primera Guerra Mundial, en 1913, apareció su trabajo fundamental, Allgemeine Psychopathologie, traducida cincuenta años más tarde al español y al inglés (1913; 1955; 1963). Esta escuela se llamó la escuela de los fenomenólogos, quienes criticaban a Kraepelin por el hecho de que este concediera igual importancia a todos los signos de una enfermedad. Los resultados de la investigación fenomenológica serían sistematizados poco antes de la Segunda Guerra Mundial por Kurt Schneider en sus síntomas de primer rango de la esquizofrenia. Schneider no pertenecía a la misma escuela fenomenológica, pero en sus inicios estuvo fuertemente influido por ella.

Este desarrollo positivo de la psiquiatría alemana apenas fue interrumpido por la Primera Guerra Mundial. Así, poco después de la guerra (en 1922, para ser más precisos) apareció un libro tan importante de las canteras de los psiquiatras fenomenológicos: Bildnerei der Geisteskranken, de Hans Prinzhorn (2001). Menos tomado en cuenta fue el subtítulo de esa obra: Ein Beitrag zur Psychologie und Psychopathologie der Gestaltung, que contiene el término Gestalt.

Una gran irrupción en la psiquiatría alemana se produjo con la llegada de los nazis. Un ejemplo: desde 1926 el psiquiatra germano-judío Wladimir Eliasberg había organizado congresos para psicoterapeutas de todas las corrientes. Desde 1928 hubo una sociedad correspondiente. En 1933 era presidente de ella Ernest Kretschmer, un psiquiatra sumamente importante, que trataba de vincular a los fenomenólogos con las escuelas psicoterapéuticas, y tenía muchos discípulos, en el Perú uno de los mejores: Grover Mori.

El 30 de enero de 1933 comenzó la dictadura nacionalsocialista. En abril de ese año se debía llevar a cabo el congreso anual de psicoterapeutas, esta vez bajo la dirección de Kretschmer. Por la cantidad de preinscripciones se esperaba la participación de algunos 
miles de asistentes. Todo estaba preparado. Los nazis, sin embargo, impidieron la participación de psiquiatras judíos. Poco tiempo necesitó Kretschmer para tomar la decisión de suspender el congreso poco antes de que se iniciara. El día que el cual el congreso debía comenzar presentó su renuncia a la presidencia. C. G. Jung se mostró dispuesto a asumirla, sobre lo cual se ha escrito mucho.

Ya antes de 1933 había habido una psiquiatría nazi, que sin embargo carecía de significación alguna. Pero desde dicho año fue significativa y condujo al holocausto a por lo menos cien mil pacientes psiquiátricos. Las teorías psiquiátricas que estaban detrás de ella eran la teoría racial y hereditaria de la esquizofrenia. Se quería limpiar a la raza alemana de la esquizofrenia.

El siglo de la psiquiatría alemana sin embargo no terminó. Por el contrario, cientos de psiquiatras judíos emigraron, en especial a Gran Bretaña y los estados donde fueron profesores de la siguiente generación de psiquiatras. En lo que concierne a Estados Unidos es conocido que en los años cincuenta los psicoanalistas alemanes ejercían un absoluto dominio en la psiquiatría norteamericana. La relación de nombres es muy importante pero también muy larga como para incluirla acá, razón por la cual solo mencionaremos algunos: Karen Horney, Kurt Goldstein, Heinz Kohut, Fritz Perls, Margaret Mahler, Frieda Fromm-Reichman, Otto Rank,
Theodor Reik. Menos conocidos son Otto Kernberg y Peter Gay, el biógrafo de Freud, si bien Kernberg emigró a Chile a los once años y Peter Fröhlich, como se llamó Gay hasta 1943, emigró a Cuba aún en la adolescencia, con 17 años, ambos fueron influidos por la escuela alemana. No debemos contar, sin embargo, a Thomas Szasz, que hasta sus 18 años habló sobre todo alemán y después fue psicoanalista, pero su formación científica la obtuvo en Estados Unidos.

En Inglaterra los psicoanalistas en torno a las dos escuelas enemigas, la de Anna Freud y la de Melanie Klein, no tuvieron influencia alguna en la psiquiatría inglesa. Por el contrario, Willi Mayer-Gross, uno de los más destacados integrantes de la escuela fenomenológica, con el apoyo de Martin Roth y E. Slater, escribió un texto de psiquiatría que fue el más importante no solo en el mundo de habla inglesa (Mayer-Gross et al., 1954). Erwin Stengel ganó -como presidente del Royal College of Psychiatrists (desde 1971 tomó ese nombre) - una gran influencia organizativa.

La preponderancia de la psiquiatría alemana en otros idiomas llegó a su fin en las postrimerías del siglo XX. El año 1980 es una fecha importante, porque fue entonces cuando se publicó el DSMIII, que rompió con la tradición y se extendió por toda la psiquiatría. Debemos renunciar a tratar por qué el DSMIII ha ganado tal influencia. Úni- 
camente diremos que en el mundo hay solo dos libros psiquiátricos con un tiraje que ha superado la frontera de los millones. El primero es el libro de Viktor Frankl (1946/1947), y el segundo el DSMIII/IV, un libro sin autor, el libro de una organización.

La diferencia esencial entre el DSMIII/IV y la psiquiatría previa consiste en seguir un ideal fisicalista, según el cual los síntomas conductuales son los que deben ser considerados como señales de la persona con trastornos, no el pensamiento, no el sentimiento ni la introspección. La cantidad de entidades psiquiátricas, de las cuales había habido solo una docena, y los criterios de determinación han alcanzado cifras astronómicas. En la actualidad hay alrededor de 935 diagnósticos propuestos por el DSM, para los cuales hay que determinar alrededor de mil criterios.

Después de la Segunda Guerra Mundial, la psiquiatría alemana dentro de Alemania se caracterizó por otras cosas, no por una renovación psiquiátrica. Más bien hubo un renacimiento de la entre tanto denominada psiquiatría alemana clásica, como también del psicoanálisis y otras escuelas psicoterapéuticas. La ruptura con la propia tradición psiquiátrica ocurrió en 1991 y fue total desde 1999. En 1991 aparece la psiquiatría estadounidense en la forma de ICD-10 como traducción alemana en un pequeño libro. Desde 1999 una nueva ley prescribe que en el cam- po de la seguridad social solo se puede emplear el ICD-10. Médico que no lo hace no recibe el pago que le corresponde por su consulta. El hecho de que la seguridad social determina el $95 \%$ de la psiquiatría práctica, ha llevado a que la mayoría de los psiquiatras alemanes crean que la psiquiatría alemana que ellos conocieron de 1899 a 1999 , es decir exactamente un siglo, está ahora algo así como prohibida. De esta manera, Alemania se ha convertido en el único país en el mundo que ha abandonado de manera radical su propia y rica tradición psiquiátrica a favor de la psiquiatría norteamericana.

Sin embargo, en Alemania este hecho no es algo excepcional. Lo podemos encontrar también en otras áreas del saber. Mientras que nadie se sorprende que químicos, físicos y biólogos alemanes prefieran publicar en inglés, sí llama la atención que lo mismo suceda con la germanística alemana. Un fenómeno como este, que abarca a tantas disciplinas, demanda algún tipo de interpretación. El motivo más importante debe ser buscado en el hecho de que hace pocos años una nueva generación se ha confrontado de manera informada y detenida con la realidad espantosa que significó el Holocausto causado por los nazis. Esta generación ha identificado a la generación de sus padres como la culpable de lo sucedido. Recién en los últimos años aparecen los primeros libros que tratan de aclarar lo que realmente sucedió. Un 
ejemplo es el libro autobiográfico de la conocida actriz y cantante alemana Wibke Bruhns (2004). Su padre fue uno de aquellos que trató de eliminar en 1944 a Hitler, razón por la cual fue llevado a la horca. Pero su padre había sido durante largo tiempo un nazi. Por qué escribió ese libro lo aclara ella misma con una sola frase cuyo sentido es fácil de entender: "Verstehen will ich, wie entstanden ist, was meine, die Generation der Nachgeborenen so beschädigt hat". [Quiero entender cómo es que surgió aquello que ha causado tanto daño a mi generación.]

\section{REFERENCIAS}

Bolten, J. Ch. (1751). Gedancken von psychologischen Cur. Halle: Hemmerde.

Brentano, F. v. (1874). Psychologie vom empirischen Standpunkt. Leipzig: Duncker \& Humblot.

Bruhns, W. (2004). Meines Vaters Land. Geschichte einer deutschen Familie. Munich: Econ.

Frankl, V. E. (1946/1947). Ein Psycholog erlebt das Konzentrationslager. Viena: Jugend und Volk.

Freud, S. (1900/1968). La interpretación de los sueños. Madrid: Biblioteca Nueva.
Herz, M. (1805). Etwas PsychologischMedizinisches: Moritz Krankengeschichte. Journal der practischen Arzneykunde und Wundarzneykunst, 5, 3-73.

Jaspers, K. (1913). Allgemeine Psychopathologie. Berlín: Springer.

Jaspers, K. (1955). Psicopatología general. Buenos Aires: Bini.

Jaspers, K. (1963). General psychopathology. Chicago: Chicago University Press.

Kant, I. (1980). Anthropologie in pragmatischer Hinsicht (7. ${ }^{\mathrm{a}}$ ed.). Hamburgo: Meiner.

Kraepelin, E. (1899). Psychiatrie. Ein Lehrbuch (6. ${ }^{\mathrm{a}}$ ed.). Leipzig: Barth.

Mayer-Gross, W.; Slater, E. \& Roth, M. (1954). Textbook of clinical psychiatry. Londres: Cassell.

Moritz, Ph. (1998). Anton Reiser. Ein psychologischer Roman. Frankfurt: Insel.

Peters, U. H. (2002a). Max IsserlinKantianer in Königsberg, Psychotherapeut bei Kraepelin, Begründer der Münchner Kinderpychiatrie, Emigrant in England. Fortschritte der Neurologie-Psychiatrie, 70 (1), 18-26.

Peters, U. H. (2002b). Die Isserlin Affäre. Ein Stellvertreter-Disput zwischen Kraepelin und Freud. Fortschritte der Neurologie-Psychiatrie, 70 (1), 27-33. 
Prinzhorn, H. (2001). Bildnerei des Geisteskranken. Ein Beitrag zur Psychologie und Psychopathologie der Gestaltung (6. ed). Viena-Nueva York: Springer.

Weikard, M. A. (1798-1799). Der philosophische Arzt. Frankfurt: Andreae.
Wolff, Ch. (1713). Vernunftigen Gedancken Von den Kräfften des menschlichen Verstandes Und Ihrem richtigen Gebrauche in Erkäntnis der Wahrheit. Halle.

Wundt, W. (1874). Grundzüge der physiologischen Psychologie. Leipzig: Engelmann. 